\title{
Telerehabilitation System in Nursing Post Stroke - A Systematic Review
}

\section{Dimas Hadi Prayoga, Bernadetta Germia Aridamayanti, Ida Trisnawati and Maria fransiska Ronalia}

Faculty of Nursing, Universitas Airlangga, Surabaya, Indonesia

\begin{abstract}
Background: Stroke is currently the main cause of disability worldwide Telerehabilitation is a rehabilitation approach that can be used to repair the damage to motor, sensory and cognitive functions. This can also substantially reduce the travel time for the health workers and therefore increase the number of patients who are consulted in a day.

Method: A search using specific keywords was done in both the journal articles and in the database. In total, 119 articles were from Scopus, 45 articles were from PubMed, 87 articles were from Science Direct, 52 articles were from EBSCO and 97 articles were from Proquest.

Result: There were 15 articles that matched the inclusion criteria. Most of the available literature only refers to short-term results and most of the research is of low quality. Evidence of the relative effectiveness was found in the types of videos, VR, cellphones and computer use. The location of the network support and technological progress are still obstacles that must be addressed immediately. Based on the current scientific evidence, only a few telerehabilitation methods can be recommended for wider use. The rehabilitation to a safe and proper condition for the sufferers due to telerehabilitation can encourage the patients to stay in rehabilitation care at home so then the patients do not need to leave home to do the rehabilitation. This can cause long-term disruption.
\end{abstract}

Conclusion: The telerehabilitation system can provide long-term therapy, meet the patient needs and save the health resources needed to minimize the severity of the patients' condition. The application of telerehabilitation is used by the nurses to optimize their role in improving patient health and telerehabilitation will answer the problem of penetrating the distance dimension.

\section{ARTICLE HISTORY}

Received: Dec 26, 2019

Accepted: Dec 31, 2019

\section{KEYWORDS}

Telerehabilitation system; nursing; post stroke; rehabilitation; long-term therapy

\section{CONTACT}

Dimas Hadi Prayoga

$\triangle$ dimas.hadi.prayoga-

2018@fkp.unair.ac.id

$\fallingdotseq$ Faculty of Nursing, Universitas

Airlangga, Surabaya, Indonesia

Cite this as: Prayoga, D. H, Aridamayanti, B.G, trisnawati, I, \& Ronalia, M. F. (2019). Telerehabilitation System in Nursing Post Stroke - A Systematic Review. Jurnal Ners, 14(3si), 182-187.

doi:http://dx.doi.org/10.20473/jn.v14i3(si).17017

\section{INTRODUCTION}

heart disease and cancer. Every year, 15 million people worldwide suffer from a stroke. Telerehabilitation is one of the most important factors that must be considered as an effort to achieve the goals tailored to the needs of the patients (DaSilva et al., 2018). The impact that can occur in poststroke patients include damage to their motor function (joint contractures, the subluxation of the shoulder joint, syndrome shoulder and the dropping of the leg), in addition to their sensory and cognitive function [10]. Another impact that also occurs in poststroke patients is aphasia (Da-Silva et al., 2018).

The merger between the use of technology and remote communication is appropriate to change conventional rehabilitation into more modern rehabilitation. Rehabilitation alternatives using telerehabilitation enable the stroke survivors to undergo physical therapy exercises without being accompanied by a therapist as they under their guidance remotely through a consultant. As a result, telerehabilitation can encourage the patients to stick with the treatment rehabilitation and it can help them to improve the recovery of their physical function due 
to the stroke having resulted in a long-term disorder that can interfere with the patient's daily activities (Yamamoto, 2017).

The telerehabilitation program (TR) was carried out to improve the patient's daily life activities and to minimize the dependence of the stroke patients. Telerehabilitation provides a strategy for the rehabilitation, evaluation and supporting strategies that improve the patient's capacity from other parties. Telerehabilitation shows evidence of the satisfaction and acceptance of the patients, their families and health workers (Sarfo et al., 2018). Telerehabilitation is a rehabilitation service for patients using information and communication technology that can be carried out by the patients from afar, This can substantially reduce the travel time for the health workers and therefore increase the number of patients who they can consult in a day (Da-Silva et al, 2018). The use of information technology in rehabilitation care can help the patients with their needs and allow them to provide care for their own patients (Sarfo et al., 2018).

\section{MATERIALS AND METHODS}

The search strategy relevant to the topic of the study was done using a database search. The databases used were Scopus, PubMed, Science Direct, EBSCO and Pro Quest. The keywords used were "stroke", "telerehabilitation", "telemedicine" and "online counseling". Full text articles and abstracts were reviewed to choose a theme that fits the criteria. The articles or journals used a randomized controlled trial. The minimum intervention duration was one month. The journal articles were published between January 2015 and September 2018. The language of the journals was English.

\section{Selection Criteria}

The PICO framework (population, intervention, control, outcome) was designed and its elements were used as the selection criteria. Articles were deemed potentially eligible if they met all of the criteria. The respondents in this study were diagnosed post-stroke as being either ischemic or hemorrhagic for $<24$ months with deficits in their motor, sensory, cognitive and function. The interventions related to the criteria for inclusion in the literature review were that they were telerehabilitation (video, virtual reality, robotics, SMS, mobile phones, computers and video games).

\subsection{Selection Method}

We examine each independently titles and abstracts of potentially eligible reports using Endnote. A total of 400 articles found, came from five databases: 119 articles in Scopus, 45 articles in PubMed, 87 article in Science Direct, 52 articles in the EBSCO, 97 articles in ProQuest. The results of the selection were in accordance with the inclusion criteria. In total, 15 articles were found and then they were given a serial number. Then the articles were analyzed. There are seven media types used in telerehabilitation, namely video, virtual reality,

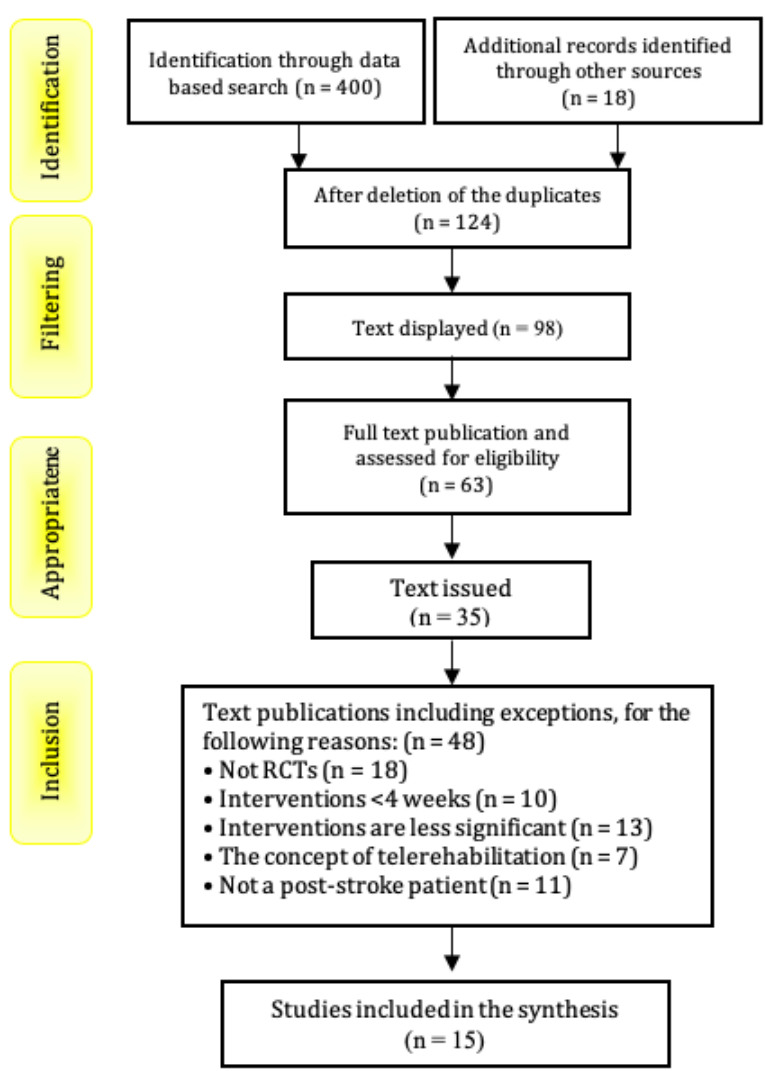

robotics, SMS, phones, computers and video games. All of the research articles were prepared and used a Randomized Controlled Trial (RCT).

\section{Data Extraction}

In this systematic review, the studies eligible for inclusion were if they tested telerehabilitation for the recovery of motor function (measured by means of different scale) in patients affected by any kind of disturbance or disease. The data extraction and management were recorded in a predesigned form with the details of the study as follows; the details of the citations that include the title, author and year of publication, the participants who met the inclusion and exclusion criteria that had been set, the participant characteristics including age, gender, the location of the stroke, time since stroke onset and their level of disability, the quality of the methodology and the telerehabilitation intervention including a description of the action procedures, the medical personnel involved, the duration of the intervention, the dose and the comparison intervention. The results of the interventions included the measures that were used and by whom, when they were given and how they were provided (directly or through information and communication technology).

\section{Data Collection and Analysis}

We filter out the article title and abstract of notes taken from the search strategy and selection criteria applying predetermined. The full text of the notes that may qualify were taken and analyzed for inclusion in this systematic review. Any disagreements can be resolved through discussion. We will extract the data from the studies and incorporate them into a 
summary table. The experimental intervention and control participants have been reported in detail.

\section{Quality Assessment}

The quality assessment study focused on areas that may overestimate the effectiveness of telerehabilitation. The quality of the randomized controlled trials varies. Unclear randomization procedures might raise questions about a potential selection bias. It provides a greater transparency than the previous approach. There is a constant uncertainty and large variations in practice, about how to assess potential biases in this particular domain of experimentation, including how to summarize biased assessments across the domains and how to incorporate biased assessments into the meta-analysis processing. The reliability of the tool has not been studied extensively, although it has been observed that there is a greater risk of bias compared to a low risk bias study.

\section{Studies Selection}

The search through the database found 400 articles from the five databases: 119 articles in Scopus, 45 articles in PubMed, 87 articles in Science Direct, 52 articles in the EBSCO and 97 articles in ProQuest. Moreover, the information was also added through the records identified through other sources. In the filtering stage, there was the removal of the duplicate articles totaling 124 articles. The text displayed in accordance with the searched keywords totaled 98 articles. Then the researcher proceeded to determine the full text and they assessed the results for eligibility, totaling 63 articles. The results of the selection in accordance with the inclusion criteria resulted in 15 articles, which were then given a serial number. There were seven media forms used in telerehabilitation, namely video, virtual reality, robotics, SMS, phones, computers and video games. All of the research articles used Randomized Controlled Trials (RCTs).

\section{RESULT}

\section{Characteristics of the Included Studies}

The total respondents in this review were 1,432 people with post-stroke complications that were either ischemic or hemorrhagic suchas hemiplagia, hemiparesis, cognitive barriers, aphasia and depression. Several studies have been conducted to identify telerehabilitation as one of the best interventions based on high technology. Examples include the use of telemedia video, virtual reality, robotics, SMS, mobile phone, computers and video games or any combination thereof. This systematic review will compare each intervention used.

\section{Telerehabilitation Post-Stroke Patients}

Telerehabilitation refers to the delivery of rehabilitation services through information and communication technologies. Clinically, this term covers a wide range of rehabilitation and telerehabilitation services that include assessment, monitoring, prevention, intervention, supervision, education, consultation and counseling. The telerehabilitation services provided to adults and children by various professionals may include but are not limited to physical therapists, speech-language pathologists, occupational therapists, audiologists, rehabilitation physicians and nurses, rehabilitation engineers, technology assistants, teachers, psychologists and dieticians. The other personnel such as the professionals, family members and caregivers can help during the telerehabilitation sessions. For the purpose of this document, the term 'professional' will be used to refer to the telerehabilitation professional service providers. The term 'client' is used to refer to all recipients of the telerehabilitation services and it is meant to include both the patients in medical settings, and the children and adults who receive the services outside of the medical sphere, for example, in school or at home(Caves et al., 2015).

Telerehabilitation has the capacity to provide services throughout the patient's lifetime and in all care settings. The point of service may include health care settings, clinics, home, school, workplace or it may be community-based. The terminology used to describe telerehabilitation is also coextensive. Some of the terms are used specifically to refer to individual rehabilitation disciplines, such as telespeech (speech language pathology) and tele OT (occupational therapy). More general terms, such as teletherapy and telepractice are also used, allowing for a broader focus on populations and activities, such as setting up education and health promotion in addition to rehabilitation. It is not the intention of this document to resolve a debate about terminology. Rather, its purpose is to provide consistency across the applications, regardless of the vocabulary used. For the purpose of this document, the term 'telerehabilitation' will be used and the reader is reminded that the terminology may vary according to the application and location(Linder et al., 2015).

The total respondents in this review consisted of 1,432 people with post-stroke complications such as eitherischemia or that were hemorrhagic like hemiplagia, hemiparesis, cognitive barriers, aphasia and depression. The necessary interventions are continuous effective to alleviate the workload of health workers. Several studies have been conducted to identify telerehabilitation as one of the best interventions based on high technology. Examples include the use of telemedia videos, virtual reality, robotics, SMS, mobile phones, computers and video games or any combination thereof. This systematic review will discuss the comparison of each intervention telemedia used(Da-Silva, Moore, \& Price, 2018).

\section{Video}

From the 15 articles, there were 4 articles that used SMS reminders as their intervention of choice. Speech therapy intervention through video is more effective at improving the process of speech and the 
pronunciation of words in post-stroke patients with aphasia (Øra et al., 2018), A similar study also explained that with the help of telerehabilitation using video, not only the patients themselves but also their families and caregivers can facilitate effective communication. Video can also better the adherence to exercise and it can allow for the remote control of the therapist(Caves et al., 2015).

\section{Virtual Reality}

Modality therapy with motor rehabilitation encourages the participation and motivation of patients with upper limb weakness in the virtual reality (E.-K. Kim et al., 2014)(J. Kim et al., 2016) using VR as an intervention for patients with upper extremity hemiparesis. The patients were trained in the supination and pronation of the forearm, extension and flexion of the elbow and shoulder, abduction and adduction, internal rotation and ekstrenal shoulder using the application of "guru virtual" and by giving visual feedback both positive and negative. This provides online information about the quality and performance of the patient's motor skills. A similar study using VR recording through the Jintronix system was used to monitor the progress of the patient's exercise (Hernandez et al., 2015).

\section{Robot}

This intervention was carried out by Linder M. et al. and Fuentes et al. It used HMP (Hand Mentor Pro) using a pneumatic pump to facilitate the active assisted movement of the wrist and fingers. Each participant using the robot's movements will have their results recorded into a computer. Telerehabilitation with robotics is a good intervention and it has become a practical approach to provide post-stroke care with limited resources. However, the results of Chen et al's study explain that the home-based rehabilitation telesupervising is as effective as conventional outpatient rehabilitation at improving the functional recovery of stroke patients. It can ease the burden of the families and caregivers of patients during the treatment(Jin et al., 2015; Linder et al., 2015).

\section{SMS, Mobile Phone and Computer}

Some interventions combine telemedia, which is SMSMobile and Mobile-Computers. Sarfo et al. used an intervention device UA-767 Plus Bluetooth and smartphones to monitor and report the measurement of blood pressure and the patient's compliance with medication schedules. The patient takes the medication for 3 months and then gives feedback. Other interventions include a Mobile-based Computer-aided Prevention System (CAPSYS) control used to monitor blood pressure and the risk of stroke in patients. The system uses text to speech software (TTS) in CAPSYS to read the blood pressure and to provide direct feedback. The system lifestyle in the form of computerized telephone-based coaching can support the usual treatment in reducing the risk factors of cerebro-cardiovascular damage (Sarfo et al., 2018; Spassova, Vittore, Droste, \& Rösch, 2016).

\section{Video Game}

Motor interventions, such as Constraint-Induced Movement therapy (CI Therapy)=based Video Game Outpatient Rehabilitation for Stroke (Vigorous), increase the intensity of training and the techniques to carry-over post-stroke patients and to improve their brain repair. However, this approach is less intense and less effective if used as a clinical treatment. In addition, many barriers to access to care are due to differences in the cognitive differences of each patient when getting the intervention (Gauthier et al., 2017).

\section{DISCUSSION}

The results of this systematic review and analysis show that the use of telerehabilitation has a significant influence on the process of long-term care in stroke patients. In practice, the treatment of stroke patients in the outpatient unit is very difficult to facilitate especially for people who live in areas that are far outside of urban areas. The existence of the current inpatient rehabilitation and outpatient care should be sustainable. Many forms telerehabilitation as a form of post-stroke intervention may show promising results (van de Ven, Schmand, Groet, Veltman, \& Murre, 2015). By using the telerehabilitation system, it is possible to provide rehabilitation services at the patient's home or in the form of community-based technology, allowing the healthcare professionals to monitor a patient's health status and to identify the conditions that require improvement before ill effects occur (J. Kim et al., 2016; van de Ven et al., 2015).

Telerehabilitation involves the provision of rehabilitation through information and communication technologies. Thus it involves the role of nurses as educators, collaborators and consultants to provide nursing care through high-tech intervention requires interdisciplinary collaboration between different health professionals. On the other hand, it is easy for the nurses do a "home care" visit albeit with limited resources.

From the review of the articles as done by some of the researchers, there are several drawbacks that may occur in the application of telerehabilitation in patients post-stroke. Telerehabilitation enables for remote communication and the use of tools. The use of technology in telerehabilitation can be applied to countries that are still experiencing keterbelakangaan. However, some of them still lack the support of the internet and a good signal for accessing the telerehabilitation itself. The level of a country's economy is also very influential in the implementation of the technology-based rehabilitation model. Prolonged use will also impose a small cost, so individual economic factors also become an obstacle to implementing the rehabilitation using telerehabilitation. Studies have 
been done that indicate that there are limitations related to the lack of knowledge of the use of tools used by the post-stroke patients in the rehabilitation process, hence this can affect the outcome and use of telerehabilitation in post-stroke patients. For further research, it is advised to provide complete information on how to use the rehabilitation equipment for both medical personnel and the patients so as to not cause bias in the research.

\section{CONCLUSION}

This systematic review does not provide final evidence about the efficacy of telerehabilitation in the recovery of the patient's motor function. The rehabilitation in this systematic review refers to the form of the clinical application of therapeutic services including counseling, prevention and diagnostics through two-way interactive telecommunications technology. This intervention provides an alternative to routine outpatient rehabilitation services such as post-stroke patients with motor, sensory and psychiatric barriers in the form of video, virtual reality, robotics, SMS, telephone, computers and video games. This intervention is also an alternative to the rehabilitation method of "home care", which requires a therapist, nurse or doctor who needs to make a trip to the patient's home. The rehabilitation of a safe and appropriate condition for the patients due to telerehabilitation can encourage the patients to stay on the course of rehabilitation care at their home. This means that the patients do not need to leave home to do the rehabilitation which can cause long-term disruption. However, the application of telerehabilitation must consider increasing the workload of the nurses in each country's national health system. In addition, there needs to be support from all parties in the development of innovative internet-based rehabilitation service infrastructure, trial use and an analysis of the cost-effectiveness is also needed.

\section{REFERENCES}

Caves, K., Png, C., Tay, A., Kumar, Y., Koh, Y. S., Chen, C., ... Hoenig, H. (2015). Singapore Tele-technology Aided Rehabilitation in Stroke (STARS) trial: protocol of a randomized clinical trial on telerehabilitation for stroke patients. BMC Neurology, 15(1), 1-14. https://doi.org/10.1186/s12883015-0420-3

Da-Silva, R. H., Moore, S. A., \& Price, C. I. (2018). Selfdirected therapy programmes for arm rehabilitation after stroke: a systematic review. Clinical Rehabilitation, 32(8), 1022-1036. https://doi.org/10.1177/0269215518775170

Gauthier, L. V., Kane, C., Borstad, A., Strahl, N., Uswatte, G., Taub, E., ... Mark, V. (2017). Video Game Rehabilitation for Outpatient Stroke (VIGoROUS): Protocol for a multi-center comparative effectiveness trial of in-home gamified constraint-induced movement therapy for rehabilitation of chronic upper extremity hemiparesis. BMC Neurology, 17(1), 1-18. https://doi.org/10.1186/s12883-017-0888-0

Hernandez, A., Kaizer, F., Raz, A., Kairy, D., Veras, M., Archambault, P., ... Levin, M. F. (2015). Maximizing post-stroke upper limb rehabilitation using a novel telerehabilitation interactive virtual reality system in the patient's home: study protocol of a randomized clinical trial. Contemporary Clinical Trials, 47, 49-53. https://doi.org/10.1016/j.cct.2015.12.006

Jin, W., Chen, J., Shi, F., Yang, W., Zhang, Y., Liu, Y., ... Ren, C. (2015). Home-based tele-supervising rehabilitation for brain infarction patients (HTRBIP): Study protocol for a randomized controlled trial. Trials, 16(1), 1-8. https://doi.org/10.1186/s13063-015-0585-5

Kim, E.-K., Jang, S.-H., Choi, Y.-H., Lee, K.-S., Kim, Y.-J., Kim, S.-H., \& Lee, H.-K. (2014). Effect of an Oral Hygienic Care Program for Stroke Patients in the Intensive Care Unit. Yonsei Medical Journal, 55(1), 240. https://doi.org/10.3349/ymj.2014.55.1.240

Kim, J., Pyun, S.-B., Eun, S.-D., Yoon, B., Lee, M., \& Son, J. (2016). Comparison of individualized virtual reality- and group-based rehabilitation in older adults with chronic stroke in community settings: a pilot randomized controlled trial. European Journal of Integrative Medicine, 8(5), 738-746. https://doi.org/10.1016/j.eujim.2016.08.166

Linder, S. M., Rosenfeldt, A. B., Bay, R. C., Sahu, K., Wolf, S. L., \& Alberts, J. L. (2015). Improving quality of life and depression after stroke through telerehabilitation. American Journal of Occupational Therapy, 69(2), 1-11. https://doi.org/10.5014/ajot.2015.014498

Øra, H. P., Kirmess, M., Brady, M. C., Winsnes, I. E., Hansen, S. M., \& Becker, F. (2018). Telerehabilitation for aphasia - Protocol of a pragmatic, exploratory, pilot randomized controlled trial. Trials, 19(1), 1-10. https://doi.org/10.1186/s13063-018-2588-5

Sarfo, F., Treiber, F., Gebregziabher, M., Adamu, S., Patel, S., Nichols, M., ... Ovbiagele, B. (2018). PINGS (Phone-based intervention under nurse guidance after stroke) interim results of a pilot randomized controlled trial. Stroke, 49(1), 236-239. https://doi.org/10.1161/STROKEAHA.117.0195 91

Spassova, L., Vittore, D., Droste, D. W., \& Rösch, N. (2016). Randomised controlled trial to evaluate the efficacy and usability of a computerised phone-based lifestyle coaching system for primary and secondary prevention of stroke. $B M C$ Neurology, 16(1), 1-9. https://doi.org/10.1186/s12883-016-0540-4

van de Ven, R. M., Schmand, B., Groet, E., Veltman, D. J., \& Murre, J. M. J. (2015). The effect of computerbased cognitive flexibility training on recovery of executive function after stroke: Rationale, design and methods of the TAPASS study. BMC Neurology, 15(1), 1-12. https://doi.org/10.1186/s12883015-0397-y 
JURNAL NERS

Yamamoto, Y. (2017). Health Informatics. https://doi.org/10.1177/1460458212462077 